\title{
L'importance de l'expertise locale dans la gestion concertée de la faune sauvage. Quels enseignements des guides de chasse de l'office National des Forêts pour les associations communales de chasse?
}

The importance of the local expertise in the joint management of the wildlife. What teachings of the hunting guides of the Forests National Office for the municipal hunter's associations?

\section{François Saldaqui}

\section{(2) OpenEdition}

\section{Journals}

Édition électronique

URL : http://journals.openedition.org/economierurale/3358

DOI : 10.4000/economierurale.3358

ISSN : 2105-2581

Éditeur

Société Française d'Économie Rurale (SFER)

Édition imprimée

Date de publication : 1 mars 2012

Pagination : 65-78

ISSN : 0013-0559

Référence électronique

François Saldaqui, «L'importance de l'expertise locale dans la gestion concertée de la faune sauvage. Quels enseignements des guides de chasse de l'Office National des Forêts pour les associations communales de chasse ? », Économie rurale [En ligne], 327-328 I janvier-mars 2012, mis en ligne le 01 mars 2014, consulté le 10 mai 2019. URL : http://journals.openedition.org/economierurale/3358 DOI : 10.4000/economierurale.3358 


\title{
L'importance de l'expertise locale dans la gestion concertée de la faune sauvage Quels enseignements des guides de chasse de l'Office National des Forêts pour les associations communales de chasse?
}

\author{
François SALDAQUI • Université de Pau et des Pays de l'Adour, Laboratoire Société Environ- \\ nement Territoire, UMR 5603 CNRS \\ francois.saldaqui@gmail.com
}

L

expansion démographique et géogra- phique des populations de grands ongulés sauvages au sein des massifs forestiers, en plaine comme en montagne (Baubet, 1998), est une réalité en France (Poinsot, 2008) et dans de nombreux pays européens (Apollonio et al., 2010). Si la situation satisfait $a$ priori chasseurs et gestionnaires d'espaces protégés, elle soulève différents problèmes relatifs à la régulation de leurs effectifs. L'augmentation des dégâts agricoles et du montant de leur indemnisation à la charge des chasseurs ${ }^{1}$, renforcée par la récente extension, même partielle, de ces mesures aux sylviculteurs (Charlez, 2008), engendre une multiplication des conflits entre gestionnaires de la faune, agriculteurs et sylviculteurs (Bages et Nevers, 1982). Ces derniers, grâce à leurs actions de mise en valeur des milieux (Arnould et Glon, 2005) peuvent être considérés comme des producteurs de gibier (Bages et Nevers, op. cit. ; Bozon et al., 1981). S'ils concèdent à ce titre subir des dégâts, leur importance et surtout leur rémanence les mécontentent fortement. Ils reprochent aux chasseurs de limiter leurs

1. Depuis cinq ans, le montant total des indemnisations des dégâts agricoles fluctue entre 20 et 23 millions d'euros à l'échelle française (Guibert, 2008). À titre d'exemple, pour le seul département des Landes, le montant des dégâts de sangliers indemnisés est passé de $100000 €$ en 1998 à $500000 €$ en 2007. prélèvements, facilitant ainsi l'augmentation des populations. De fait, si les débats se cristallisent sur la question des dégâts, c'est plutôt celle de la maîtrise des effectifs des populations qui est en jeu, la réduction des premiers ne pouvant être atteinte durablement ${ }^{2}$ que par celle des seconds. L'estimation des niveaux de prélèvements permettant de satisfaire les intérêts opposés des chasseurs et des producteurs, comme les conditions de leur réalisation, sont donc au cœur des débats. Il s'agit de s'accorder, en fonction de la sensibilité des milieux aux déprédations, sur une densité d'animaux présents sur le territoire suffisamment élevée pour assurer des prélèvements « abondants » mais assez faible pour limiter au maximum les dégâts. Ce niveau qui doit correspondre à un « équilibre agro-sylvocynégétique » (Normant et al., 2004), est présenté comme l'objectif vers lequel doivent tendre les gestionnaires. Il se révèle pourtant éminemment subjectif et sujet à de nombreuses variations compte tenu de trois facteurs essentiels : l'évolution des milieux, les niveaux d'effectifs et les intérêts économiques en jeu. Il apparaît qu' «en réalité la

2. La mise en œuvre de mesures destinées à prévenir l'émergence des dégâts (clôtures électriques, aménagements du milieu ou même agrainage et affouragement) ne permettent que de limiter les dégâts mais ne traitent pas la source du problème, les niveaux de populations. 
forêt est écartelée au gré des propriétaires entre fonction cynégétique et fonction sylvicole » (Corvol, 2004). Pour tenter de composer avec ces fonctions et intérêts opposés (Lorgnier du Mesnil, 2003), des instances de négociations ouvertes aux représentants de chaque partie existent. En leur sein, ils tentent de dégager un consensus sur le niveau de population « optimum » à conserver.

Toutefois, ces négociations supposent en amont une bonne connaissance des effectifs (Granjou et Mauz, 2009) qui, dans les faits, est généralement très partielle. Or, la détermination du plan de chasse (Fol et Tassel, 1966), nombre d'animaux à abattre en une année le plus souvent à l'échelle communale, repose sur leur évaluation précise. Les comptages permettant leur mesure présentant des taux d'incertitude parfois élevés (Smart et al., 2004 ; Boussin, 2006 ; Morellet et al., 2007 ; Bertouille, 2008), ils engendrent souvent des plans de chasse trop faibles, favorisant l'essor des populations, notamment des cervidés (Normant et al., op. cit.) S' accorder sur un niveau de prélèvements satisfaisant à la fois les intérêts cynégétiques et économiques sans connaître précisément la population de départ apparaît donc complexe (Morellet, 2008).

À ces enjeux relatifs à l'évaluation des populations, s'ajoutent ceux liés à la configuration écologique du territoire de gestion sur lequel la répartition des animaux est aussi irrégulière que celle des couverts végétaux sensibles (jeunes parcelles forestières, cultures de maïs...) Dans ce cadre, considérer que la réalisation «mécanique » du plan de chasse sur le terrain satisfera tous les protagonistes, ouvre la porte à de nombreux malentendus. Idéalement, il doit être mis en œuvre en des lieux et sur des animaux précis (Servanty et al., 2008), voire même aux moments des déprédations les plus aiguës. Pour la gestion forestière, compte tenu de la temporalité spécifique de la sylviculture, les objectifs de gestion doivent en outre intégrer une importante vision prospective (Klein et al., 2008).

La conception d'un plan de chasse dénué d'enjeux territorialisés plus précis que ceux relatifs à la limite administrative du territoire auquel il s'applique montre ici ses limites et explique en partie les tensions entre chasseurs et producteurs. Dès lors, si la difficulté de mettre en œuvre une régulation efficace des grands ongulés sur un territoire résulte de l'ambition de concilier des objectifs différents, elle suppose en amont une connaissance fiable des niveaux de populations et des caractéristiques des milieux.

La clé de cette gestion réside principalement dans la maîtrise par les gestionnaires locaux de ces divers paramètres. Cette situation privilégiée n'est pourtant pas courante en France où si le droit de chasse est lié à la propriété (Chamboredon, 1982 ; Charlez, 1997), il revient aux chasseurs de gérer la faune sur un foncier qu'ils ne possèdent et n'exploitent pas. Dans cette configuration, ils ne maitrisent qu'une partie des paramètres nécessaires. Or, dans une logique d'efficacité, le plan de chasse se doit d'intégrer ces deux dimensions.

Pourtant, de telles organisations ne sont pas inconcevables ${ }^{3}$. Parmi ces gestionnaires, maitrisant à la fois la gestion des couverts végétaux et celle des grands ongulés, l'Office national des forêts (ONF), dans les forêts domaniales constitue un acteur particulièrement intéressant à étudier. D'abord parce que la production forestière constitue a priori son objectif premier, ensuite parce qu'il met en œuvre cette gestion « hybride » dans des situations territoriales variées.

3. Dans les chasses privées, les propriétaires se réservent le droit de chasse qu'ils valorisent au même titre que la production forestière. Une démarche similaire se retrouve dans les forêts domaniales gérées par des guides de chasse, voire dans certaines réserves nationales de chasse et de faune sauvage où des régulations sont mises en œuvre à l'image de celles des Bauges et d'Orlu). 
«En définitive, ce système ne fonctionne que si le forestier est un peu chasseur et le chasseur quelque peu forestier!» (Gourmain, 2004). Étudier la manière dont l'établissement parvient, par l'intermédiaire d'agents reconnus pour leurs compétences cynégétiques, les guides de chasse, à définir et à atteindre cet « équilibre », alors qu'il délègue assez largement, dans un souci déjà ancien de valorisation économique (Fol, 1961 ; Estève, 2004) la régulation des cheptels, constitue l'objet de cet article. Examiner comment, dans un contexte dénué de toute « opposition chasseurs/producteurs », les opérateurs parviennent à concilier ces intérêts opposés, peut fournir des éléments de solution transposables aux structures communales de chasse.

Notre analyse s'insère dans le cadre d'un travail de thèse concernant les conditions et contraintes avec lesquelles les gestionnaires locaux de la faune doivent composer dans leur pratique quotidienne. Ce travail accorde une large place à l'étude des territoires de gestion

envisagés dans leur diversité. Diversité de leurs statuts d'une part avec des espaces protégés, des espaces urbains et des « campagnes banales » mais également des enjeux qui s'y superposent : socio-économiques (dégâts, vente de «produits chasse », productions agricoles et sylvicoles), écologiques ou encore cynégétiques. Diversité des gestionnaires (fonctionnaires, bénévoles ou propriétaires fonciers) d'autre part, qui œuvrent au sein de territoires dont les superficies, les formes, la proximité de centres urbains ou encore les modes de mise en valeur des sols définissent à chaque fois des contextes spécifiques de gestion. Il s'agit donc dans chaque territoire étudié d'analyser les éléments qui facilitent ou contraignent la mise en œuvre d'une gestion efficace des grands ongulés.

L'enquête conduite auprès des guides de chasse ONF, des fédérations départementales des chasseurs des Landes et d'Ariège mais également chasseurs en forêts doma- niales ${ }^{4}$ à pour but, dans le contexte particulier de l'Office, de définir les conditions d'émergence d'une personnalité forte de la gestion de la faune, l'expert local, référent tant au niveau de ses connaissances cynégétiques que relationnelles. Elle a pour cadre des forêts domaniales dans lesquelles la place de l'agent dans l'expertise ou l'accompagnement des chasseurs varie fortement en fonction des enjeux locaux, allant d'une totale main mise sur le territoire à un « simple» avis consultatif.

C'est donc par l'examen détaillé du rôle des guides de chasse au sein de leur territoire d'action, que nous préciserons les conditions par lesquelles l'Office parvient à concilier sylviculture et régulation cynégétique. L'approche territoriale de cette gestion s'avère alors déterminante puisque « l'équilibre sylvo-cynégétique » tant recherché dépend de la sensibilité des milieux, et donc des productions, aux atteintes des espèces, elle-même déterminée par la nature ou la fonction des couverts forestiers.

Avant d'entrer dans l'examen détaillé de ce fonctionnement précisons les conditions dans lesquelles l'ONF organise la pratique cynégétique dans les forêts domaniales.

\section{La gestion des forêts domaniales sous contraintes économiques et territoriales}

L'Office est représenté sur le terrain par des agents exerçant dans une ou plusieurs forêts. Autrement appelées "lots de chasse », elles constituent des territoires de gestion à part entière dotés d'un locataire, d'un règlement et d'un plan de chasse spécifiques. Certaines d'entre elles font l'objet d'un découpage en plusieurs lots, alors gérés chacun de manière indépendante. Dans tous

4. Soit une quinzaine d'entretiens réalisés en 2007 et 2008 pour cette étude et complétée dans le cadre du travail de thèse par d'autres menés au sein de territoires communaux de chasse et espaces protégés. 
les cas, les conditions de gestion d'un lot par l'agent local sont partiellement liées aux contraintes que font peser ces forêts sur les territoires communaux où elles se trouvent.:

Trois cas de figure se présentent. Lorsque le massif forestier a pour vocation première la protection (d'un milieu littoral, de versants montagneux), la production sylvicole est réduite. Valoriser le gibier par une chasse accompagnée très rémunératrice est alors souvent pratiqué par l'Office. Lorsque de «petites forêts » domaniales se trouvent isolées au milieu de vastes étendues relevant du droit privé, elles ne restreignent que modérément l'accès des chasseurs locaux au territoire de chasse. Dans ce cas, l'ONF exploite directement le gibier sous forme d'adjudications au plus offrant. À l'opposé, lorsque l'emprise domaniale couvre de vastes portions de territoire communal, elle prive les associations locales de territoires de chasse significatifs. Dans ce cas, c'est par le biais d'amodiations, locations à tarifs modérés, que le droit de chasse est cédé aux associations locales. L'agent est alors moins investi dans le suivi de la pratique cynégétique.

Comme bien des propriétaires forestiers privés, l'ONF « moderne » (devenu Établissement public à caractère industriel et commercial donc tenu d'autofinancer ses activités), perçoit donc son patrimoine comme doublement productif : il rapporte par la production forestière mais aussi par le gibier qui s'y trouve. Les modalités de valorisation de ce dernier relèvent donc de trois statuts réglementaires.

On les examinera du plus libéral, pour lequel le temps consacré à la garderie par les agents est faible, au plus encadré, nécessitant une surveillance étroite de l'action de chasse très prenante mais compensée par une valorisation accrue du gibier.

\section{Les adjudications publiques}

Ce mode de fonctionnement consiste en une contractualisation du droit de chasse sous forme d'adjudications publiques au plus offrant, validées par des baux conclus pour une durée de 6 ou 12 ans : l'ONF délègue ici totalement son droit de chasse. Les agents ne sont donc pas présents sur le terrain durant l'action de chasse, mais définissent le plan de chasse en accord avec les locataires et contrôlent les prélèvements. Ce système nécessitant une faible présence de l'agent local est de fait relativement lucratif pour l'établissement. Largement utilisé dans le nord de la France, où les pratiques de cession du droit de chasse au plus offrant sont courantes, il est plus rare au sud où par tradition, le gibier est une ressource libre d'accès et la chasse un exercice peu réglementé (Gény-Mothe, 2003).

\section{Les amodiations aux ACCA locales}

Les amodiations correspondent à des locations de gré à gré à des Associations communales de chasse agrée locales (ACCA). D'un fonctionnement voisin du précédent, ce modèle s'en différencie par un coût des locations relativement peu élevé. Cette concession vise à maintenir un territoire de chasse suffisant aux ACCA dont une large part est couverte de forêts domaniales. Bien que moins lucrative pour l'ONF, cette forme de cession du droit de chasse offre l'avantage « de conserver la paix sociale $»^{5}$. Dans ce système, comme dans le précédent, la part de liberté laissée aux chasseurs est importante. Toutefois, en échange de la liberté concédée pour un coût modéré, l'établissement attend une réalisation efficace des plans de chasse. Dans le cas contraire, l'agent local peut intervenir directement dans la gestion des prélèvements, ou même les assurer avec le concours de collègues.

\section{Les licences individuelles ou collectives}

La vente de licences exige une présence accrue de l'agent local. Selon le degré d'implication des agents dans l'organisation et

5. Formulation reprise à plusieurs occasions par les guides de chasse et certains chasseurs locaux au sujet des amodiations. 
l'accompagnement de la chasse, l'ONF accorde trois types de licences à la journée $^{6}:$ les licences de chasse organisée, les licences de chasse dirigée, les licences de chasse guidée (d'après ONF : www.onf.fr, 2008).

L'ONF conserve dans ce cas son droit de chasse et gère directement la production et les prélèvements. Le plus souvent privilégiées par des «touristes cynégétiques », ces prestations offrent l'avantage d'une gestion fine des prises. La présence permanente d'un agent ONF sur le domaine et dans l'accompagnement de la chasse permet en effet de réaliser des prélèvements efficaces en prévention des dégâts. En regard de la sensibilité des parcelles aux déprédations, il détermine les individus à abattre, les lieux et parfois même la période où doit se réaliser la chasse. Avec ce mode de gestion, les compétences de l'agent s'avèrent déterminantes dans la performance du système. Il peut alors pratiquer une gestion des cheptels visant un équilibre sylvo-cynégétique afin de minimiser les dégâts. Bien que d'un coût en personnel élevé, ce dispositif tient sa rentabilité de la valorisation optimale qu'il permet à la fois du potentiel cynégétique et de celui de la forêt. Son objectif consiste en effet à maintenir des populations assurant des revenus élevés tout en minimisant les risques de déprédations.

$\mathrm{Si}$ au plan juridique ces trois modes de valorisation de la ressource cynégétique sont clairement distincts, dans la pratique, ils sont souvent combinés. Étudier le rôle de l'agent local et les conditions territoriales dans lesquelles il décline ces systèmes pour optimiser cette gestion

6. Les licences de chasse organisée : l'agent détermine les lieux et périodes de chasse de même que le type de gibier recherché mais est absent lors de son déroulement ; les licences de chasse dirigée, le plus souvent collectives, la direction de chasse est assurée par un agent ; les licences de chasse guidée, l'agent décide des lieux, périodes et des animaux qui sont prélevés et guide le chasseur. (d'après ONF : www.onf.fr, 2 octobre 2008)
" sylvo-cynégétique » constitue le cœur de cette analyse. On examinera pour ce faire quatre forêts domaniales landaises, girondines et ariégeoises (figure 1), qui présentent chacune des particularités du point de vue des modes de gestion comme des contraintes sociales ou réglementaires dans lesquelles la régulation s'opère.

Figure 1. Localisation des forêts domaniales étudiées

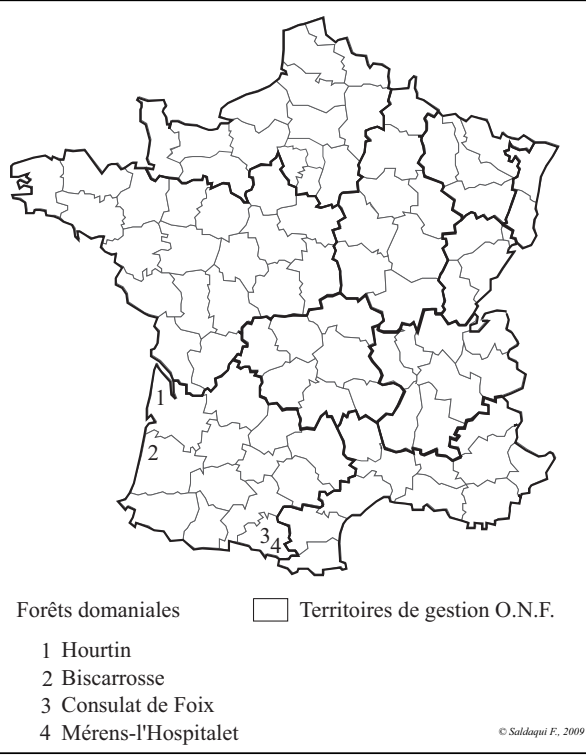

Source : d'après O.N.F.

\section{La régulation sous garderie étroite des ongulés en forêt landaise}

L'analyse des deux forêts landaises et girondines permet d'examiner comment une gestion à la fois fine du point de vue de la maîtrise des dégâts, et pourtant rémunératrice pour l'organisme, est possible par le biais de licences individuelles et collectives à Hourtin et seulement collectives à Biscarrosse.

\section{Hourtin \\ Une forêt sous haute surveillance}

La première d'entre elles, située à Hourtin en Gironde, est caractérisée par la présence permanente d'un agent ONF, reconnu comme guide de chasse, qui permet un encadrement strict de la pratique cynégétique. 
Figure 2. La forêt domaniale d'Hourtin en Gironde, cadre d'une pratique cynégétique strictement encadrée (d'après IGN)

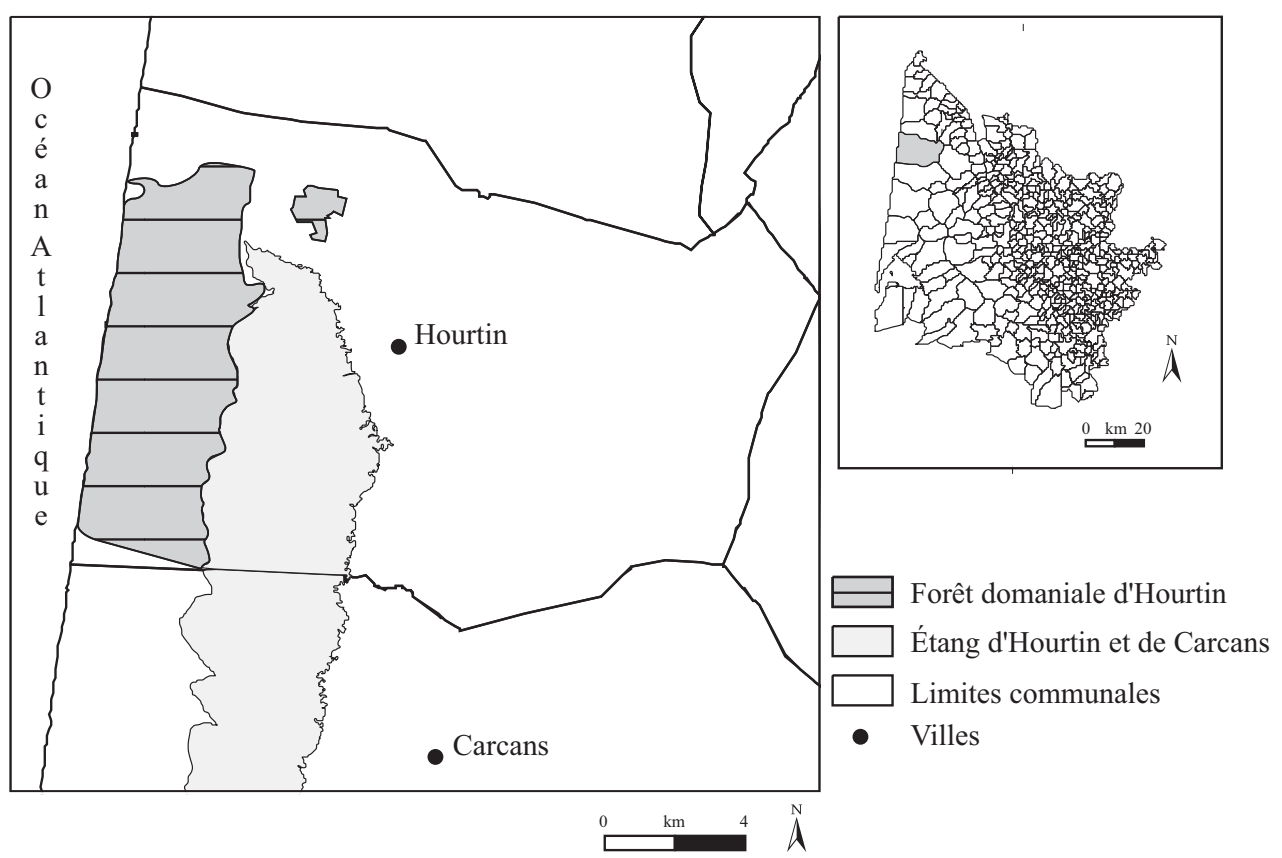

(C) Saldaqui F, 2009

Bordée à l'ouest par l'océan Atlantique et à l'est par l'étang d'Hourtin, ce massif d'une superficie de 4000 hectares, majoritairement composé de pins maritimes (figure 2), attire de nombreux chasseurs « étrangers », notamment bordelais, en quête de territoires de chasse (Menautl, 1994), la proximité de grandes villes permettant de pratiquer des tarifs plus élevés. Ceci permet une valorisation accrue du gibier, rendue possible par la faible emprise de la forêt domaniale sur le territoire communal (environ $20 \%$ ). La superficie modeste de ce massif facilite, par opposition à des territoires de gestion beaucoup plus vastes, sa bonne connaissance par l'agent local.

Bien que peu productive du point de vue sylvicole, cette forêt de protection littorale exploitée comporte des parcelles à divers stades de développement, et donc de vulnérabilité variable. La vente d'un «produit chasse » représente dans ce contexte un double avantage : compenser la faible productivité du couvert forestier et prévenir l'émergence de dégâts.
Ayant une connaissance globale mais également fine de «sa » forêt, l'agent, à partir de la mosaïque des états de maturité du peuplement, planifie et encadre les prélèvements de manière à préserver les parcelles les plus sensibles aux déprédations par une surchasse localisée, tandis que d'autres, faisant office de refuge, sont exemptes de prélèvement (figure 3).

Les coupes de bois sont planifiées de façon à ne pas amoindrir la capacité d'accueil du milieu qui engendrerait soit une baisse des populations et donc de la ressource en gibier, soit une accentuation des déprédations. $\mathrm{Ce}$ pilotage fin de la gestion territoriale du gibier n'est possible qu'au moyen d'une chasse guidée dans laquelle les animaux et le lieu des prélèvements sont soigneusement déterminés. La gestion du cheptel est ici totalement maîtrisée puisque 20 chevreuils, 40 sangliers et 2/3 cerfs sont annuellement prélevés.

La performance du système repose sur la double compétence du guide de chasse à la fois gestionnaire du foncier et gestionnaire du 


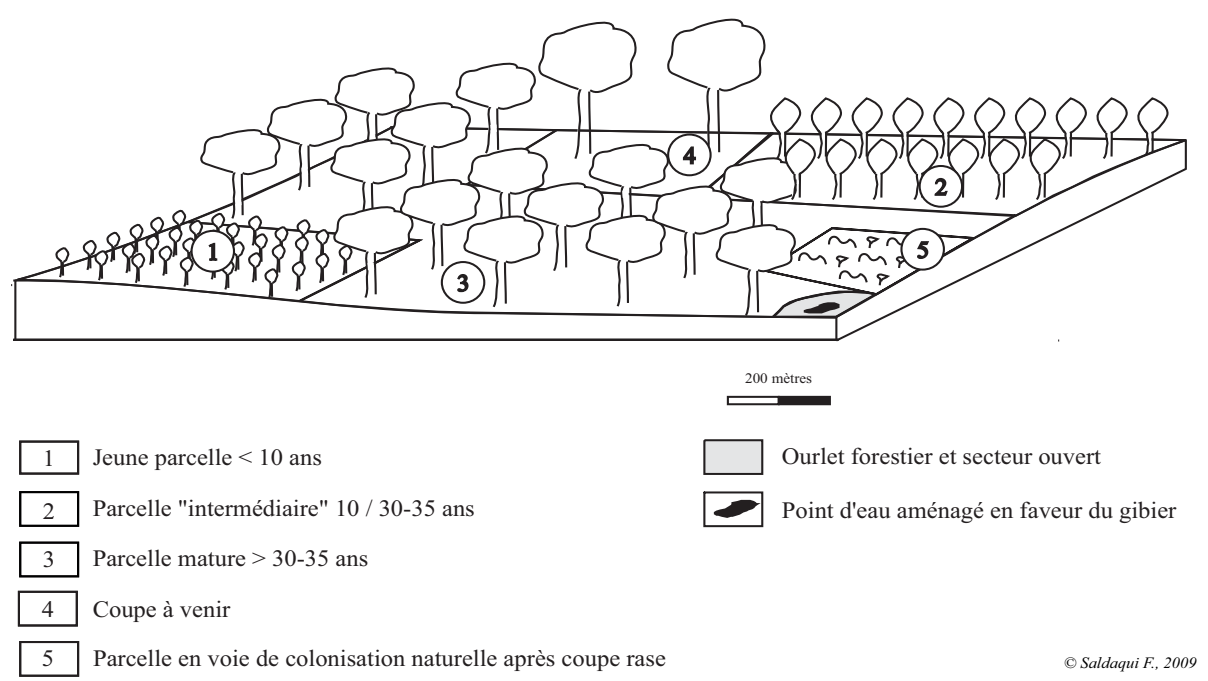

Note: Les prélèvements interviennent en priorité à proximité des parcelles sensibles aux dégâts (1), tandis que sur les moins vulnérables ils sont réalisés indifféremment $(2,3$ et 4$)$ et se font plus rares sur les parcelles « refuges » (5).

gibier qui facilite son expertise et lui confère donc la possibilité dans « sa » forêt d'adapter quantitativement et qualitativement les prélèvements aux contraintes qu'impose la présence de grands ongulés sur un espace de production.

\section{Biscarrosse}

\section{Un encadrement strict des chasseurs dans un territoire sensible}

Plus au sud, dans le département des Landes, la forêt domaniale de Biscarrosse, qui couvre 11800 hectares, est caractérisée par la présence d'un site militaire dans lequel la régulation des populations est assurée par l'ONF. Cette particularité soumet l'établissement à une réglementation limitant considérablement les conditions d'accès au territoire. En effet, pour un nombre de battues qui doit rester modeste ${ }^{7}$, l'armée décide des

7. Le statut particulier de ce territoire, centre d'essais d'armement, impose pour des raisons de sécurité de limiter la présence de civils dans l'enceinte, et occasionnellement l'accès à certains secteurs. Ces deux contraintes exigent un nombre limité de battues nécessaires à la régulation des ongulés présents sur l'ensemble du centre. lieux et dates, laissant à l'ONF le soin d'encadrer les licences collectives. Dans ces conditions, l'efficacité dans la réalisation des battues (annuellement 2 pour le chevreuil et $5 / 6$ pour le sanglier) est primordiale. Pour chacune d'elles, une soixantaine de chasseurs ${ }^{8}$ est donc nécessaire. Cet effectif important permet, d'une part d'enclore un secteur de chasse assez vaste pour déloger un maximum d'animaux. Il permet, d'autre part, de constituer une ligne de tireurs suffisamment fournie pour limiter les possibilités de fuite des individus en assurant "l'étanchéité » du dispositif (Poinsot et Saldaqui, 2009). Le contrôle renforcé des chasseurs est assuré par quatre agents de l'ONF, chacun ayant en charge un quart des participants'. Il permet alors un contrôle efficace des prises et impose un bon respect des règles propres à l'enceinte.

Investi d'une mission de police, l'Office met plus en avant ici la fonction de garde de

8. Effectif relativement élevé par rapport à certaines équipes communales restreintes à $15 / 20$ chasseurs 9. Soit environ 3 fois plus qu'en l'absence de contraintes de ce type. 
ses agents que leur capacité d'expertise territoriale, si déterminante à Hourtin. $\mathrm{Ce}$ territoire au statut réglementaire particulier exige des prélèvements efficaces, mais sous des contraintes de dates et de lieux précis. $\mathrm{Si}$ ces régulations étaient réalisées par les agents ONF eux-mêmes, elles représenteraient pour l'Office un coût élevé pour une valorisation nulle, tandis que les licences collectives engendrent une ressource financière significative. L'organisme choisit donc de recourir aux chasseurs locaux dont le nombre élevé permet des prélèvements effi- caces sous contrôle des agents ONF (à l'image de ce qui se pratique dans les « zones interdites à la chasse » du Parc national des Cévennes). Si ces pratiques sont justifiées par le statut particulier du territoire, hors de l'enceinte militaire, la réalisation du plan de chasse est confiée à l'ACCA locale, sous le régime de l'amodiation. C'est donc qu'en dehors de ces zones à statut particulier, l'ONF développe une troisième forme de gestion du gibier bien plus libérale que les deux premières, largement répandue en Ariège.

\section{La régulation cogérée des populations en forêt ariégeoise}

Figure 4. Les forêts domaniales du Consulat de Foix et Mérens-I'Hospitalet en Ariège, de vastes massifs cadre d'une pratique cynégétique en faveur des ACCA locales (d'après IGN)

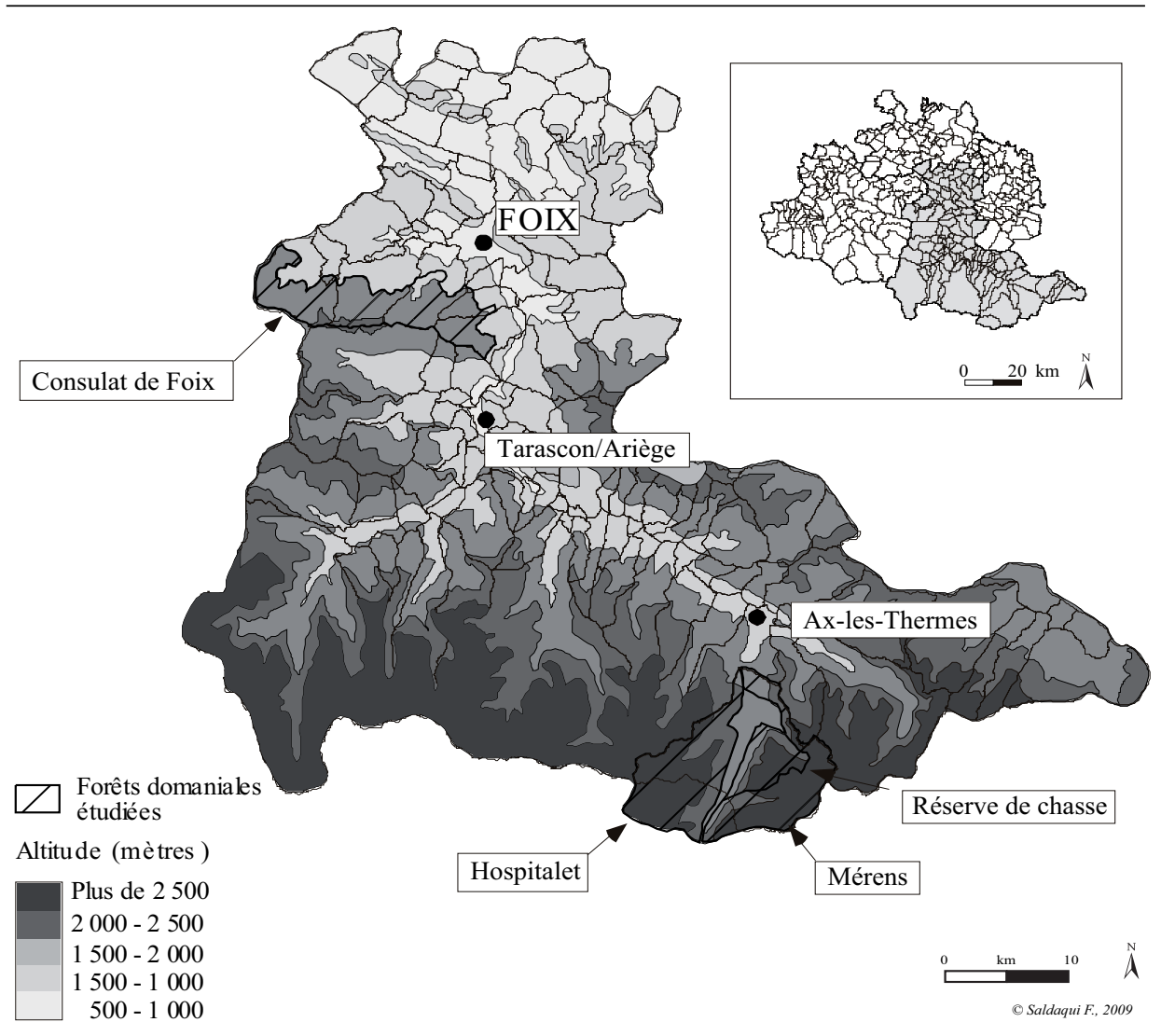

L'étude de deux forêts ariégeoises, Mérens les Vals et l'Hospitalet et Consulat de Foix (figure 4) révèle comment une gestion conjointe des effectifs de grands ongulés sous le régime d'amodiation permet de concilier une pratique de chasse locale et des impératifs économiques. 


\section{Mérens les Vals et l'Hospitalet Un contrôle a posteriori des prélèvements}

Les forêts domaniales de Mérens les Vals et de l'Hospitalet, limitrophes de la principauté d'Andorre, jouent un rôle important dans la protection contre les avalanches. Ces terrains d'altitude, aux versants escarpés, comprennent en majorité des estives et des zones rocheuses plus favorables à la chasse de l'isard qu'à celle du cerf, « limitée » aux zones forestières (figure 5).

Figure 5. La forêt domaniale de Mérens-Hospitalet au cœur d'enjeux sociaux et cynégétiques majeurs (d'après IGN)

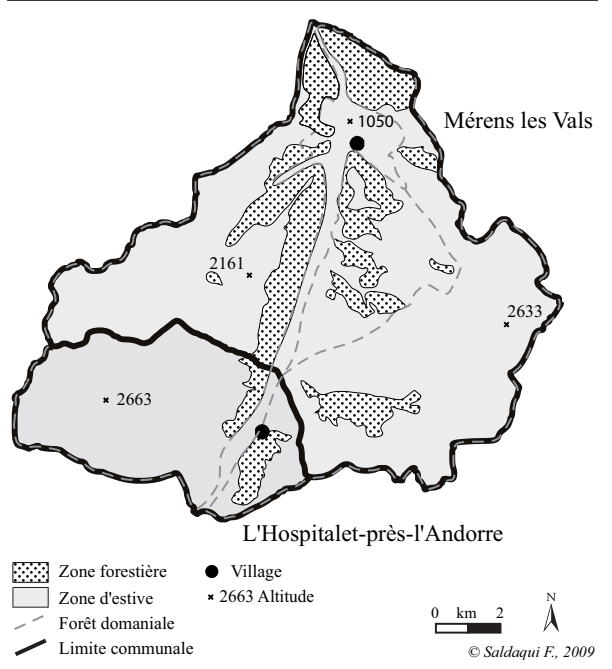

La présence de ces deux espèces permet à l'ONF d'instaurer une gestion combinant l'amodiation pour l'isard et le cerf, peu coûteuse en temps de travail pour les agents, à une chasse guidée exclusivement à l'isard, plus rémunératrice, semblable à celle pratiquée à Hourtin. Cette combinaison s'explique par le souci du gestionnaire de rentabiliser au maximum le territoire sans augmenter le loyer de l'amodiation ${ }^{10}$.

10. Dans ce cas, l'agent ONF réserve pour la chasse guidée une partie du plan de chasse alloué à l'ACCA et le réalise sur une portion du territoire à laquelle les chasseurs locaux ne peuvent accéder.
En effet, le seul loyer payé par les deux ACCA locales réunies en Association intercommunale de chasse agrée (AICA) ${ }^{11}$, ne valorise pas «suffisamment» la ressource cynégétique du massif. La solution consistant à chercher une rémunération maximale par le biais d'adjudications risquerait de priver les locaux de tout territoire de chasse, réactivant d'anciennes tensions entre l'ONF et les sociétés locales. Le recours à la chasse à l'approche permet alors à l'établissement de combler ce manque à gagner, tout en assurant aux locaux une présence durable sur le territoire. Dans ce dispositif, l'agent local est investi d'une double mission : économique, auprès des chasseurs étrangers grâce aux tirs à l'approche et gestionnaire, liée à l'estimation des cheptels pour les chasseurs locaux.

Pourtant, des difficultés de gestion du couvert forestier à forts enjeux l'ont conduit à intervenir de manière plus directe dans la gestion de l'AICA. Surplombant le village de l'Hospitalet, une forêt de protection joue un rôle essentiel dans la prévention des avalanches (figure 5). Servant de zone refuge en période hivernale pour les cerfs, elle est soumise à de nombreuses déprédations qui menacent ainsi son efficacité. La régulation du cheptel constitue donc un enjeu majeur très localisé. Or, constatant que les chasseurs locaux ne remplissaient qu'imparfaitement les objectifs du plan de chasse, l'agent local s'est vu dans l'obligation d'intervenir personnellement. Il s'est ainsi chargé de la réalisation des tirs « à titre d'exemple » durant une année, montrant ainsi la faisabilité d'une régulation « chirurgicale ». Face à cette démonstration, les chasseurs locaux ont donc repris en main les prélèvements mais sous la surveillance

11. Les AICA sont des regroupements intercommunaux de territoires de chasse destinés à rationaliser la pratique cynégétique. Le droit de chasse appartient alors à l'AICA et l'ensemble des sociétaires des communes qui la constituent ont la possibilité de fréquenter indifféremment l'ensemble du territoire ainsi créé. 
stricte de l'agent qui contrôle les prises en fin de chasse.

Dans le « rapport de force » ainsi instauré, ce n'est plus tant la qualité de l'expertise territoriale ou la fonction de police de l'agent qui importe, mais sa compétence cynégétique. En effet, en réalisant des prélèvements que les chasseurs locaux peinaient à assurer, il démontre que leur intervention manque d'efficacité. Bénéficiant de conditions financières favorables, ceux-ci se voient rappeler que leur gestion du territoire relève d'une faveur de l'Office, qui, en l'absence d'une efficacité régulatrice suffisante, pourrait envisager de mettre fin à ce régime préférentiel.

Si dans ce cas, motivé par des enjeux environnementaux, l'agent doit intervenir ponctuellement auprès des chasseurs locaux afin d'optimiser la régulation des populations, ce type d'intervention est inutile dans les territoires plus communs. C'est alors par son niveau d'expertise qu'il intervient dans la gestion des cheptels locaux.

\section{Consulat de Foix, l'expertise territoriale au service d'une pratique autonome et efficace des chasseurs}

La forêt domaniale du Consulat de Foix est louée par amodiation à une AICA réunissant six communes concernées par l'emprise du massif (figure 4). Cette forêt, gérée comme précédemment sur un principe de partage de la ressource en gibier, ici du cerf, voit se dérouler une évaluation conjointe des niveaux de population sous la forme de comptages opérés par les chasseurs locaux sous le contrôle du garde. S'y ajoutent des observations ponctuelles réalisées par les chasseurs lors de sorties ultérieures qui, rapportées au garde, permettent d'affiner la connaissance du cheptel. Ce travail de terrain offre l'avantage d'un suivi en temps réel des populations.

Il a permis, quelques années en arrière, qu'une augmentation subite du taux d'abroutissement sur de jeunes hêtres, essence normalement peu attractive pour le cerf, soit rapidement résolue. Perçue comme un « signal d'alarme », témoignant d'une augmentation du cheptel (Ningre et Picard, 2006), cette hausse brutale des déprédations a vu chasseurs locaux et agent ONF s'accorder pour augmenter le plan de chasse de 65 à 80 individus. Au bout de trois années, la disparition des déprédations et le recul du nombre de cerfs ont attesté la réussite de l'opération. Le plan de chasse fut alors réduit et stabilisé entre 60 et 65 prélèvements.

Les rapports de confiance nés de cette co-évaluation de la ressource permettent que les chasseurs locaux et l'agent ONF se partagent la réalisation du plan de chasse et du territoire comme précédemment. Dans un souci d'optimisation économique, l'agent local procède à des chasses à l'approche au cerf durant la période du brame. Il conserve pour ce faire la jouissance exclusive d'une partie du territoire pour quelques semaines avant d'en restituer l'usage aux locaux pour le reste de la saison ${ }^{12}$.

Dans ce contexte, la confiance réciproque entre l'agent local et les chasseurs permet une cogestion efficace des populations dans un esprit de partage d'expérience apprécié pour les deux partis.

\section{Analyse et discussion}

L'étude de ces quatre terrains souligne différentes manières d'atteindre une gestion efficiente des populations de grands ongulés qui suppose en amont une bonne connaissance des niveaux de population et des enjeux locaux de leur régulation et de composer avec de nombreux aspects économiques, sociaux ou encore réglementaires, tout en déléguant pourtant assez largement l'acte de chasse. La déclinaison des modes

12. Ce partage territorial est nécessaire à la réalisation de ce type de chasse qui nécessite de s'approcher à faible distance de l'animal et donc que ce dernier ne soit pas effarouché suite à de nombreuses battues. 
de gestion et leur adaptation aux contraintes de chaque territoire permet notamment à l'Office de mettre en œuvre le partage d'une ressource entre chasseurs étrangers et locaux sans que cela ne génère de conflit.

L'examen du dispositif de gestion de grand gibier mis en œuvre par l'ONF dans ses lots de chasse permet par comparaison de révéler les difficultés rencontrées par les ACCA. L'analyse des différents terrains révèle dans un premier temps que son efficacité tient à la triple compétence de l'établissement : maîtrise foncière, maîtrise de la production forestière et enfin maîtrise de la gestion cynégétique.

Cette configuration permet à chaque guide de chasse d'ajuster (cas d'Hourtin) ou de faire correspondre (cas ariégeois) les prélèvements, tant sur le plan quantitatif que qualitatif, aux contraintes locales. Par son expertise, l'agent peut proposer une répartition spatiale et temporelle très fine du plan de chasse en fonction, de l'organisation des milieux (cas d'Hourtin et du Consulat de Foix), de localisation des parcelles sensibles (cas de Mérens et l'Hospitalet) ou encore des contraintes réglementaires (cas de Biscarosse).

Le statut même du guide est également déterminant. Outre ses fonctions d'expertise, ses attributs de «police de la chasse » au sein des forêts domaniales en font en effet l'arbitre du bon fonctionnement de la régulation selon deux principaux leviers d'action : l'un réglementaire, l'autre économique. Du point de vue réglementaire, il peut, en cas de gestion déficiente, ne pas renouveler une licence ou se charger luimême de la régulation (cas de Mérens et l'Hospitalet) privant ainsi les chasseurs d'une partie de la ressource en gibier. Il peut également être le garde classique qui encadre la réalisation du plan de chasse (cas de Biscarrosse), ou définit les modalités de sa réalisation (cas d'Hourtin). Sur le plan économique, il peut augmenter le tarif des amodiations, limitant ainsi fortement les possibilités d'accès des chasseurs locaux au territoire (recours évoqué à Mérens et 1'Hospitalet).

Ces deux aspects mettent en avant le rôle pivot de l'agent local, dont une grande partie de la légitimité réside dans son statut de fonctionnaire assermenté, aux leviers cœrcitifs potentiellement puissants. Cette situation ne peut être considérée indépendamment des enjeux financiers que sous-tend la rentabilisation des fonds domaniaux. Dès lors, le choix de l'Office d'affecter à ces postes des personnels chasseurs favorise la tenue d'une gestion efficace des grands ongulés. Ce fonctionnement reste toutefois limité aux seules forêts domaniales. Il ne peut donc en aucun cas constituer un modèle aisément transposable aux territoires communaux placés sous régime associatif ${ }^{13}$.

En effet, dans ces territoires, chasseurs et producteurs constituent deux groupes d'acteurs distincts dont les rapports au sujet de la régulation des grands ongulés sont souvent conflictuels. Ces tensions engendrées par les déprédations s'expliquent par plusieurs facteurs que l'analyse du fonctionnement de l'Office permet de révéler.

En premier lieu, la fonction de sylviculteur assignée aux guides de chasse leur confère une connaissance fine de leur territoire et des zones sensibles aux dégâts. Ceci autorise donc une estimation précise des niveaux de prélèvements à réaliser et des secteurs à privilégier dans une approche prospective du territoire très limité dans les ACCA.

En second lieu, la responsabilité financière de l'Office est très différente de celle des ACCA. Celui-ci est en effet conditionné par une logique de rentabilité (voire de mission d'utilité publique avec les forêts de protection) qu'une mauvaise gestion des

13. Au sein des territoires communaux peuvent exister des chasses privées au sein desquelles les propriétaires adoptent une logique proche de celle de l'ONF. Ils exploitent conjointement la production forestière et le grand gibier. Liés par bail aux chasseurs, ils s'assurent par le biais du levier financier, d'une gestion efficace du gibier. 
grands ongulés peut fortement compromettre. Dans les ACCA, les chasseurs, s'ils indemnisent les dégâts, ne les règlent pas directement, la tâche en incombant aux fédérations départementales de chasse. L'effet lissant et déresponsabilisant d'une telle procédure constitue une différence de taille entre les deux dispositifs expliquant pour partie quelques latitudes dans la gestion associative du grand gibier.

Enfin, grâce à sa fonction de police, l'Office s'assure du respect des engagements pris par les chasseurs. Une association communale de chasse ne dispose d'aucune réelle fonction de cet ordre. Ainsi, contraindre les adhérents à fournir un effort supplémentaire de régulation ou à prospecter un secteur précis paraît difficile. Toutefois, les responsables associatifs peuvent être ponctuellement secondés dans leur rôle par un lieutenant de louvèterie au statut de fonctionnaire bénévole disposant d'un pouvoir de police et nommé par le Préfet. Ceci concourt statutairement à le rapprocher d'un guide de chasse pour encadrer administrativement certaines interventions de régulation. Reconnu pour ses aptitudes cynégétiques et investi de l'autorité préfectorale, il peut en effet définir et imposer les conditions de réalisation des interventions de régulation (lieu, nombre de chasseurs, choix des chasseurs...).

\section{Conclusion}

Dans ce contexte, en dehors des forêts domaniales, la figure d'expert local suppose que celui-ci dispose, outre de compétences cynégétiques, d'une bonne connaissance des milieux et des enjeux et objectifs propres aux producteurs. Dans l'idéal, il est un référent et un interlocuteur privilégié auprès des producteurs permettant une réponse adaptée des chasseurs locaux à toute apparition de dégâts : battue en période de chasse ou simple déplacement en période de fermeture. Ces relations permettent la construction, si ce n'est d'un « savoir gérer » commun et partagé, au moins d'une prise de conscience mutuelle des contraintes que rencontrent producteurs et chasseurs. Ainsi, lorsqu'une personne peut et sait jouer ce rôle d'interface, voire même de médiateur, il devient un référent et réduit l'éloignement courant qui existe entre ces deux groupes. Il endosse alors un rôle d'expert local proche de celui des guides de chasse de l'Office. Il est souvent reconnu et plébiscité par les autres chasseurs qui lui confient la présidence de l'association locale. Cette fonction lui confère une place essentielle dans la mise en œuvre de la régulation des grands ongulés.

La notion d'expertise locale se révèle donc indispensable dans l'optique d'une régulation efficace du grand gibier. Si elle est basée sur la connaissance des effectifs présents sur un territoire et de leurs rapports avec les milieux, elle ne suffit pourtant pas à assurer une maîtrise « parfaite » des populations et des dégâts. Elle ne constitue qu'un aspect des conditions et contraintes territoriales avec lesquelles les chasseurs associatifs, à l'image des guides de chasse de l'Office, doivent composer dans leur gestion courante. 


\section{RÉFÉRENCES BIBLIOGRAPHIQUES}

Apollonio M., Anderson R., Putman R. (2010). European ungulates and their management in the 21 st century. Cambridge University, $604 \mathrm{p}$.

Arnould P., Glon E. (2005). Introduction. La nature a-t-elle encore une place dans les milieux géographiques, p. 7-16.

Bages R., Nevers J.-Y. (1982). L'organisation locale de la chasse. Auto-défense collective et régulation des conflits. Études rurales, ${ }^{\circ}$ 87-88, p. 209-221.

Baubet E. (1998). Biologie du sanglier en montagne : biodémographie, occupation de l'espace et régime alimentaire. Thèse de doctorat de biologie des populations, Gaillard J.-M. (dir.), Université Claude Bernard, Lyon 1, 297 p.

Bertouille S. (2008). Dynamique des populations de cerf en région wallonne. Forêt wallonne, $\mathrm{n}^{\circ} 94$, p. 56-66.

Boussin L. (2006). Société et animal : contrôler la grande faune sauvage. L'exemple du chevreuil dans le département de la Haute Vienne. Thèse de doctorat en géographie, Valades B. (dir.), Université de Limoges, 435 p.

Bozon M., Chamboredon J.-C., Fabiani J.L. (1981). Les usages sociaux du cadre naturel. Élaboration sociale et conflit des modes de consommation de la campagne : l'exemple de la chasse. Des forêts et des hommes, p. 273-279.

Corvol A. (2004). Préface. Forêt et chasse. $X^{e}-X X^{e}$ siècle. Paris, Harmattan, p. 3-14.

Chamboredon J.-C. (1982). La chasse et les usages sociaux de l'espace rural. Études rurales, ${ }^{\circ}$ 87-88, p. 233-260.

Charlez A. (1997). Le droit de chasse, le droit de propriété et l'environnement. Droits de propriété et environnement, p. 77-85.

Charlez A. (2008). Plan de chasse et dégâts à la forêt : l'évolution. Faune sauvage, $\mathrm{n}^{\circ} 281$, p. 56-63.
Estève C. (2004). La location du droit de chasse en forêt domaniale. Forêt et chasse. $X^{e}-X X^{e}$ siècle. Paris, Harmattan, p. 281-299.

Fol R. (1961). Recherche d'un équilibre entre la forêt et le gibier à Chambord. Revue forestière française, p. 815-826.

Fol R., Tassel F. (1966). Un des facteurs déterminants du plan de chasse : l'accroissement des populations de cervidés. Revue forestière française, p. 149-159.

Gény-Mothe M. (2003). Les chasses traditionnelles : des chasses populaires, patrimoine culturel des départements du SudOuest. L'homme du Midi. Sociabilité méridionales, p. 145-160.

Gourmain P. (2004). Gestion sylvicole, gestion cynégétique : quelle cohabitation ? Forêt et chasse. $X^{e}-X X^{e}$ siècle, Paris, Harmattan, p. 85-88.

Guibert B. (2008). Bilan national de l'indemnisation des dégâts de sanglier. Modalités de gestion du sanglier, Actes du colloque, Reims, $1^{\text {er }}$ et 2 mars 2007, p. 73-78.

Granjou C., Mauz I. (2009). Quand les animaux échappent à leurs traceurs. Comment les acteurs s'arrangent avec l'incertitude. p. 117-134.

Klein F., Rocquencourt A., Ballon P. (2008). Pour un meilleur équilibre sylvo-cynégétique. Des pratiques favorables aux cervidés.

Lorgnier du Mesnil. (2003). Chasse et forêt. L'équilibre forêt-gibier. Paris, Gerfaut, $136 \mathrm{p}$.

Menautl J. (1994). Chasseurs et citadins en bordelais. Les enjeux récents de la pratique d'un loisir traditionnel. Recherches urbaines, $\mathrm{n}^{\circ}$ 9, $194 \mathrm{p}$.

Morellet N. (2008). La gestion des grands herbivores par les indicateurs de changement écologique. Faune sauvage, $\mathrm{n}^{\circ} 282$, p. 9-18. 
Morellet N., Gaillard J.-M., Hewison M., Ballon P., Boscardin Y., Duncan P., Klein F., Maillard D. (2007). Indicators of ecological change: new tools for managing populations of large herbivores. Journal of Applied Ecology, $\mathrm{n}^{\circ} 44$, p. 634-643.

Ningre F., Picard J.-F. (2006). Le hêtre et les cervidés : de vrais « faux amis ». Les nouvelles de "Pro Sylva Wallonie», $\mathrm{n}^{\circ} 19$, p. 6-9.

Normant P., Ballon P., Klein F. (2004). À propos de l'équilibre sylvo-cynégétique et des moyens de l'obtenir. Rendez-vous techniques, $\mathrm{n}^{\circ} 6$, p. 14-18.

Poinsot Y. (2008). Les enjeux géographiques d'une gestion durable de la faune sauvage en France. Annales de géographie, $\mathrm{n}^{\circ} 663$, p. 26-47.

Poinsot Y., Saldaqui F. (2009). Quelle organisation territoriale pour une gestion durable des populations de sangliers ? L'exemple de l'UG 15 sur le piémont pyrénéen. Mappemonde, ${ }^{\circ} 94$.

Servanty S., Gaillard J.-M., Toïgo C., Lebreton J.-D., Baubet E., Klein F., Brandt S. (2008). Comment le prélèvement qualitatif du sanglier peut-il influencer sa démographie. Forêt wallonne, $\mathrm{n}^{\circ}$ 92, p. 6-14.

Smart J.-C.-R., Ward A.-I., White P.-C.-L. (2004). Monitoring woodland deer populations in the UK: an imprecise science. Mammal Review, $\mathrm{n}^{\circ}$ 34, p. 99-114. 